\title{
SOME CONSIDERATIONS OF MASS TRANSFER UNDER VACUUM*
}

RYOZO TOEI, MORIO OKAZAKI AND MASASHI ASAEDA

Department of Chemical Engineering, Kyoto University, Kyoto, Japan

\begin{abstract}
Mass transfer between infinite parallel plates under vacuum was treated by analyzing the Boltzmann equation with the two-sided Maxwellian velocity distribution function of molecules. The equation obtained from this analysis coincides completely with that derived from the "series-resistance model" of the resistances of evaporation, condensation and diffusion. The results were compared with the theoretical results obtained by Uyeha et al. in 1960 .
\end{abstract}

\section{Introduction}

The problem of mass transfer under vacuum was treated in 1960 , by Uyeha et al. ${ }^{1)}$ who proposed two mechanisms of mass transfer through highly rarefied stagnant gas, one of which was named "molecularprojective evaporation" and the other "molecularburst evaporation". The former is the case where molecules evaporated from the surface are transferred directly to the condensing surface without intermolecular collisions. In the latter case molecules are transferred to the condensing surface colliding with the same kind of molecules as themselves. In both cases the stagnant gas is so rarefied that its effect on mass transfer may be negligibly small. By considering the slip model of diffusion, they also treated the intermediate region which exists between the region described above and the region where the usual diffusion equation can be applied. But, in their treatment, an uncertain modification of the transfer distance was introduced which made the results unreasonable.

Here we treated the same problem of mass transfer in two ways, one of which is based on the Boltzmann equation or from a microscopic stand point, and the other is based on the macroscopic "series-resistance mode ${ }^{12)}$ with some further consideration of the boundary conditions.

\section{Theorefical Derivations}

\section{2-1 Two-sided Maxwellian model}

According to this model, the velocity distribution function of the molecules at any point between parallel plates is assumed as in Fig. 1 for each kind of molecules of $\mathrm{A}$ and $\mathrm{B}$;

* Received on November 9, 1970

$$
f_{\alpha i}=n_{\alpha i}\left(m_{\alpha} / 2 \pi k T\right)^{3 / 2} \exp \left[-\left(m_{\alpha} / 2 k T\right)\left(u^{2}+v^{2}+w^{2}\right)\right]
$$

where $i=1$ for $u>0, i=2$ for $u \leq 0$ and $\alpha=\mathrm{A}, \mathrm{B}$. Here the system is assumed to be isothermal.

Using these distribution functions, the basic Boltzmann equation can be solved by moment methods to obtain relations among $n_{\mathrm{A} 1}, n_{\mathrm{A} 2}, n_{\mathrm{B} 1}, n_{\mathrm{B} 2}$ and $x^{3}$.

The integral form of one-dimensional Boltzmann equation is

$$
\int Q u \frac{d f}{d x} d c=\int Q\left(f^{\prime} f_{1}^{\prime}-f f_{1}\right) g b d b d \varepsilon d \boldsymbol{c}_{1} d c
$$

Integrating Eq.(2) with substitution of $m_{\mathrm{A}}, m_{\mathrm{B}}, u_{\mathrm{A}} m_{A}$, $u_{\mathrm{B}} m_{\mathrm{B}}$ in $Q$, we can obtain the conservation equations: mass conservation for $\mathrm{A}$ and $\mathrm{B}$ molecules, $Q=m_{\mathrm{A}}, m_{\mathrm{B}}$

$$
\begin{aligned}
& \frac{d}{d x}\left(\frac{m_{\mathrm{A}} k T}{2 \pi}\right)^{1 / 2}\left(n_{\mathrm{A} 1}-n_{\mathrm{A} 2}\right)=0, \quad \text { or } \quad n_{\mathrm{A} 1}-n_{\mathrm{A} 2}=\gamma^{\prime} \\
& \frac{d}{d x}\left(\frac{m_{\mathrm{B}} k T}{2 \pi}\right)^{1 / 2}\left(n_{\mathrm{B} 1}-n_{\mathrm{B} 2}\right)=0, \quad \text { or } \quad n_{\mathrm{B} 1}-n_{\mathrm{B} 2}=\beta,
\end{aligned}
$$

momentum conservation for $\mathrm{A}$ and $\mathrm{B}, Q=u_{\mathrm{A}} m_{\mathrm{A}}, u_{\mathrm{B}} m_{\mathrm{B}}$

$$
\begin{aligned}
& \frac{k T}{2} \cdot \frac{d}{d x}\left(n_{\mathrm{A} 1}+n_{\mathrm{A} 2}\right)=\Delta Q_{\mathrm{AB}} \\
& \frac{k T}{2} \cdot \frac{d}{d x}\left(n_{\mathrm{B} 1}+n_{\mathrm{B} 2}\right)=\Delta Q_{\mathrm{BA}}
\end{aligned}
$$

where $\Delta Q_{\mathrm{AB}}$ is the collision integral which means the rate of exchange of the momentum between $\mathrm{A}$ and $\mathrm{B}$ molecules. Since the total momentum change of the two kinds of molecules is zero, i.e. $\Delta Q_{\mathrm{AB}}+\Delta Q_{\mathrm{BA}}=0$,

$$
\begin{aligned}
\frac{k T}{2} \cdot \frac{d}{d x}\left(n_{\mathrm{A} 1}+n_{\mathrm{A} 2}+n_{\mathrm{B} 1}+n_{\mathrm{B} 2}\right)=0 \\
\text { or } n_{\mathrm{A} 1}+n_{\mathrm{A} 2}+n_{\mathrm{B} 1}+n_{\mathrm{B} 2}=\delta^{\prime}
\end{aligned}
$$

Assuming Maxwellian molecules, $\Delta Q_{\mathrm{AB}}$ can be calculated to obtain the following result;

$$
\begin{aligned}
\Delta Q_{\mathrm{AB}} & =\int Q\left(f^{\prime} f_{1}^{\prime}-f f_{1}\right) g b d b d \varepsilon d c_{1} d c \\
& =-\frac{1}{n_{\mathrm{t}} D}\left(\frac{2 k T}{\pi m}\right)^{1 / 2}\left(\begin{array}{c}
k T \\
2
\end{array}\right) n_{\mathrm{B} 1}
\end{aligned}
$$

And the mass flux of A molecules is 


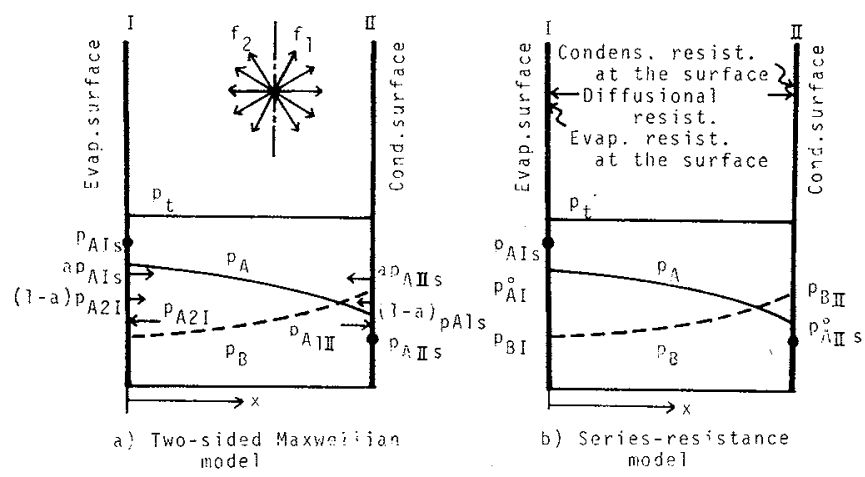

Fig. 1 Models for mass transfer

$$
N_{\mathrm{A}}=\int m_{\mathrm{A}} u_{\mathrm{A}} f_{\mathrm{A}} d \boldsymbol{c}_{\mathrm{A}}=\left(\frac{m_{\mathrm{A}} k T}{2 \pi}\right)^{1 / 2} \gamma^{\prime}
$$

Similarly the mass flux of B molecules, which is zero, is

$$
N_{\mathrm{B}}=\left(\frac{m_{\mathrm{B}} k T}{2 \pi}\right)^{1 / 2}\left(n_{\mathrm{B} 1}-n_{\mathrm{B} 2}\right)=0, \quad \text { or } \quad n_{\mathrm{B} 1}=n_{\mathrm{B} 2}
$$

Eqs. (3), (5), (7), (9) and (10) can be written as follows, using the relation $p=n k T$,

$$
\begin{aligned}
& p_{\mathrm{A} 1}-p_{\mathrm{A} 2}=\gamma \\
& \frac{d}{d x}\left(\frac{p_{\mathrm{A} 1}+p_{\mathrm{A} 2}}{2}\right)=-\zeta \gamma p_{\mathrm{B} 1} \\
& p_{\mathrm{A} 1}+p_{\mathrm{A} 2}+p_{\mathrm{B} 1}+p_{\mathrm{B} 2}=\delta \\
& N_{\mathrm{A}}=\left(\frac{m_{\mathrm{A}}}{2 \pi k T}\right)^{1 / 2} \gamma \\
& p_{\mathrm{B} 1}=p_{\mathrm{B} 2}
\end{aligned}
$$

where

$$
\zeta=\frac{1}{2 p_{\mathrm{t}} D}\left(\frac{2 k T}{\pi m_{\mathrm{A}}}\right)^{1 / 2}
$$

These equations are solved with the following boundary conditions;

$$
\left.\begin{array}{ll}
\text { at } & x=0, \quad p_{\mathrm{A} 1}=p_{\mathrm{A} 1 \mathrm{I}}=(1-a) p_{\mathrm{A} 2 \mathrm{I}}+a p_{\mathrm{AI}} \\
\text { at } & x=L, \quad p_{\mathrm{A} 2}=p_{\mathrm{A} 2 \mathrm{I}}=(1-a) p_{\mathrm{A} 1 \mathrm{I}}+a p_{\mathrm{A} I \mathrm{~s}}
\end{array}\right\}
$$

Here $(1-a) p_{\mathrm{A} 2 \mathrm{I}}$ and $(1-a) p_{\mathrm{A} 1 \mathrm{I}}$ are the pressures corresponding to the molecules rebounded at surfaces I and II respectively, and $a p_{\text {AIs }}$ and $a p_{\text {AIS }}$ are for the molecules evaporated from surfaces I and II respectively (see Fig. 1).

The result can be written with measurable values of the saturated vapor pressures of the evaporating material at the temperatures of surfaces $I$ and II, $p_{\text {AIS }}, p_{\text {AIS }}$ and the pressure of the stagnant gas at surface II, $p_{B \text { II }}$ as follows;

$$
p_{\mathrm{AIS}}-p_{\mathrm{AII}}=\frac{2-a}{a} \gamma+p_{\mathrm{BI}}\left(1-e^{-\zeta \gamma L}\right)
$$

where " $a$ " is the evaporation coefficient of the evaporating substance. We can calculate the mass flux under various driving forces and partial pressures of the stagnant gas by solving Eq. (13) for $r$ and substituting the value into Eq. $\left(9^{\prime}\right)$.

\section{2-2 Series-resistance model}

Three resistances for the mass transfer may be considered in series as in Fig. 1. They are the resistance of evaporation, the resistance of diffusion between the

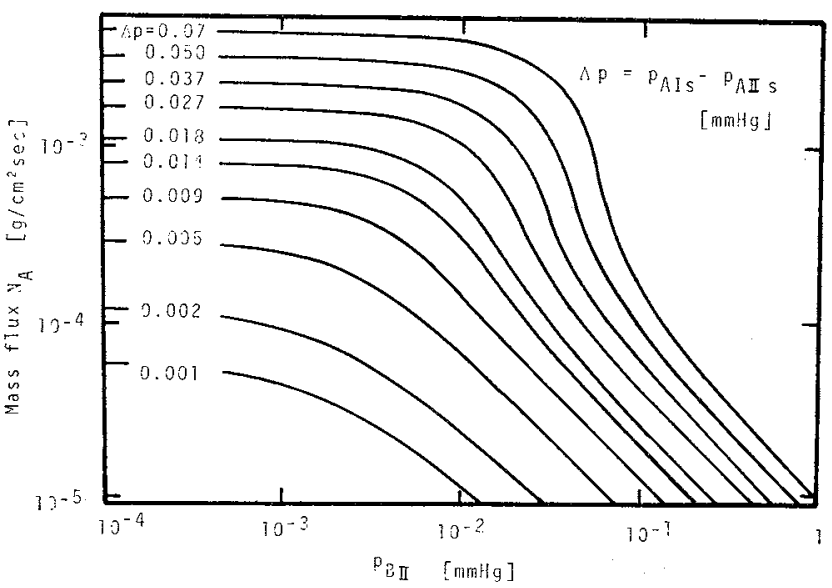

The estimated diffusion coefficient; $D=0.0356 \mathrm{~cm}^{2} /$ sec. at $0^{\circ} \mathrm{C}, 1 \mathrm{~atm}$ and the diffusion distance; $L=5.5$ $\mathrm{cm}$, evaporation coeff.; $a=1$

Fig. 2 Theoretical mass transfer rate calculated from Eqs. $\left(9^{\prime}\right)$ and (13) for di-2-ethylhexyl-phthalate

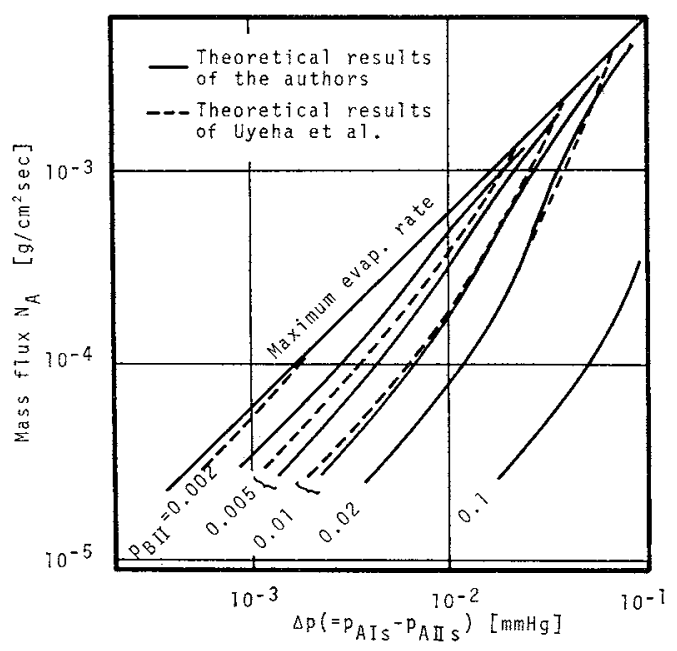

The vapor pressure of di-2-ethylhexyl-phthalate used in the calculation; $0.07 \mathrm{mmHg}$ at $150^{\circ} \mathrm{C}, 1.2 \mathrm{~mm}$ at $200^{\circ} \mathrm{C}$. $D=0.0356 \mathrm{~cm}^{2} / \mathrm{sec} . L=5.5 \mathrm{~cm}$

Fig. 3 Comparison of the theoretical results

evaporating and condensing surfaces and the resistance of condensation.

For the evaporation and condensation rates, the Hertz-Knudsen equation may be used;

$N_{\mathrm{A}}=a\left(\frac{M_{\mathrm{A}}}{2 \pi R T}\right)^{1 / 2}\left(p_{\mathrm{AIS}}-p_{\mathrm{A} 2 \mathrm{I}}\right)=a\left(\frac{M_{\mathrm{A}}}{2 \pi R T}\right)^{1 / 2}\left(p_{\mathrm{A} I \mathrm{I}}-p_{\mathrm{AIS}}\right)$

Here again the molecules are considered to be classified in two parts according to their directions of velocities, just as in section 2-1. And for the diffusion between the evaporating and condensing surfaces, the usual equation of diffusion through stagnant gas may be used;

$$
N_{\mathrm{A}}=\frac{M_{\mathrm{A}} p_{\mathrm{t}} D}{R T L} \ln \frac{p_{\mathrm{BI}}}{p_{\mathrm{BI}}}
$$

Considering that the total pressure is uniform and expressed as

$$
p_{\mathrm{t}}=p_{\mathrm{AI}}^{\circ}+p_{\mathrm{BI}}=p_{\mathrm{BII}}^{\circ}+p_{\mathrm{BI}}
$$


where

$$
\begin{aligned}
& p_{\mathrm{AI}}^{\circ}=\frac{1}{2}\left\{a p_{\mathrm{AIS}}+(1-a) p_{\mathrm{A} 2 I}+p_{\mathrm{A} 2 \mathrm{I}}\right\} \\
& p_{\mathrm{AII}}^{\circ}=\frac{1}{2}\left\{a p_{\mathrm{AIS}}+(1-a) p_{\mathrm{A} I \mathrm{I}}+p_{\mathrm{A} 1 \mathrm{I}}\right\}
\end{aligned}
$$

and using Eqs. (14) and (15), we can obtain the same equation as Eq.(13).

\section{Comparison with Other Theoretical Results}

The theoretical results for mass flux $N_{\mathrm{A}}$ are shown in Fig. 2, in case of $a=1$, against the partial pressure of the stagnant gas at the condensing surface with various driving forces $\left(\Delta p=p_{\mathrm{AIS}}-p_{\mathrm{AIS}}\right)$ as the parameter.

This figure shows that as the partial pressure of the stagnant gas $p_{\mathrm{BI}_{\mathrm{I}}}$ at the condensing surface decreases, mass flux increases and approaches the maximum value given by the Hertz-Knudsen equation, Eq.(14), with $p_{\mathrm{A} 2 \mathrm{I}}=p_{\mathrm{AIS}}$. But the situation at large driving forces is somewhat different from that at rather smaller driving forces. As $p_{\mathrm{BI}}$ decreases, the mass flux at large driving forces approaches the maximum faster than at smaller driving forces or at high $p_{\mathrm{BI}}$, a large driving force is necessary for the mass flux to approach the maximum value. Also, the mass flux approaches the maximum in a different way. These results may be understood by considering that, as the mass flux increases due to the magnitude of the driving forces or to the rarefaction of the stagnant gas, the molecules of stagnant gas are driven to the condensing surface. As a result, the number of stagnant gas molecules in the diffusing space decreases, even when $p_{\mathrm{BI}}$ remains constant. Therefore, the diffusion resistance also decreases.

These results may be compared with the theoretical results obtained by Uyeha et al., who made an analysis based on a slip-diffusion model. They proposed a model similar to that in section $2-2$, but their treatment of the boundary conditions is different from the one in this paper. They modified Eq. (15) using a parameter called the "transfer distance", $x$ ', which may be interpreted as the Knudsen layer near the evaporating surface, and $L-x^{\prime}$ instead of $L$. According to their theory, this transfer distance is related to the effective mean free path of the molecules near the evaporating surface by two different equations considering the mechanisms of mass transfer described in section 1.

In Fig. 3 the mass flux $N_{\mathrm{A}}$ calculated from Eq. (13) is compared with the one recalculated according to the theory of Uyeha et al. . As is shown in this figure, at rather high pressure of stagnant gas these two results coincide completely because of the negligibly small value of $x^{\prime}$. As the pressure decreases, however, the transfer distance $x^{\prime}$ increases due to the increase of the mean free path, and the difference between these two results becomes greater. Concerning this difference, it has been shown experimentally,4) that the theoretical results obtained from the two-sided Max- wellian model are in good accordance with the experimental results in the region of mass flux smaller than about $2 \times 10^{-4} \mathrm{~g} / \mathrm{cm}^{2} \cdot \mathrm{sec}$. Another difference is that according to Eq. (13) the mass flux approaches asymptotically the maximum evaporation rate as the driving force increases with a constant partial pressure of stagnant gas at condensing surface, while according to Uyeha's theory the mass flux coincides with the maximum evaporation rate at a definite driving force. The coincidence of the mass flux with the maximum evaporation rate in the presence of stagnant gas seems to be unreasonable because the molecules of stagnant gas will collide with the diffusing gas molecules and, therefore, some resistance must be exerted.

\section{Conclusions}

An equation which describes the mass transfer rate under vacuum was derived from the Boltzmann equation with two-sided Maxwellian velocity distribution function. The qeuation, which contains only one parameter of the evaporation coefficient, coincides with that derived from the classical seriesresistance model.

From the equation derived here the mass transfer rate between parallel plates can be calculated in the whole range of rarefaction without considering such mechanisms as proposed by Uyeha et al., whose theory contains empirical and postulated rélations to evaluate the transfer distance in each mechanism.

The mass flux calculated according to the authors' theory coincides well with that by Uyeha et al. in the range of rather high pressure of the stagnant gas, but a fairly large difference is found between them in the low-pressure range as shown in Fig. 3. Furthermore, there exists another difference between the authors' results and Uyeha's in the range of large driving force or of high mass flux; the mass flux approaches the maximum in a different way as shown in Fig. 3. Because of the reasons described in section 3, however, the results derived here seem to be more reasonable than those of Uyeha et al. .

\section{Nomenclafure}

$a=$ evaporation coefficient

$b=$ collision parameter

$D=$ diffusion coefficient

$f=$ velocity distribution function

$f^{\prime}=$ velocity distribution function for the molecule of the velocity after collision

$f_{1}=$ velocity distribution function of molecules named " 1 "

$g=$ relative speed

$k=$ Boltzmann constant

$L=$ distance between the evaporating and the condensing surfaces

$M=$ molecular weight

$m=$ weight of a molecule

$N=$ mass flux

$n=$ number density of molecules

$P=$ pressure

$u, v, w=$ velocity components in the directions of $x, y, z$, respectively

$x^{\prime}=$ transfer distance defined by Uyeha, et al. 
$\beta, \gamma, \gamma^{\prime}=$ integral constants

$\delta, \delta^{\prime}=$ integral constants

$\langle$ Suffix $\rangle$

$1,2=$ directions for $x>0$ and $x \leq 0$ respectively

$\mathrm{A}, \mathrm{B}=$ molecules of $\mathrm{A}$ and $\mathrm{B}$ respectively

I, II = at the evaporating and condensing surfaces respectively

$\mathrm{s} \quad=$ saturated value

$\mathrm{t}=$ total value
Literature cited

1) Uyeha, H., T. Kajiura and O. Yoshikawa: Kagaku Kögaku (Chem. Eng., Japan) 24, 5, 274 (1960)

2) Sherwood, T. K. and N. E. Cooke: A.I.Ch.E. Journal, $3,37(1957)$

3) Toei, R., M. Okazaki, A. Uragami and Y. Takaki: J.Ch. Eng. of Japan 1, 2, 125 (1968)

4) to be published

\title{
LONGITUDINAL DISPERSION OF GAS IN A MOVING BED AT LOW REYNOLDS NUMBERS*
}

\author{
SOK MOON YOON** AND DAIZO KUNII \\ Department of Chemical Engineering, University of Tokyo, \\ Tokyo, Japan
}

\begin{abstract}
Employing two kinds of fine glass keads at low Reynolds numbers, the longitudinal dispersion coefficients of gas were measured in a moving bed by analyzing the response curves measured at two downsiream points of a bed. The dispersion data obtained in a moving bed were comparable with the results investigated by Carberry and Bretton ${ }^{3}$ in a packed bed for the same sysiem of helium-air. The effect of solid velocities on the gas mixing was not so favorable, and the concept of tortuosity factor was acceptable to describe the discrepancy of the data with the molecular diffusivity at low Reynolds numbers.
\end{abstract}

According to the best of the authors' knowledge, studies of the mixing of fluids in moving beds are very few, and the only available data were obtained by Muchi et al. ${ }^{10}$. Applying the methods developed by Gilliland and Mason ${ }^{7,8)}$, Muchi et al. measured the longitudinal dispersion coefficients of gas and liquids. They also measured the radial dispersion coefficients of gas by the procedure given by Hanratty et al.9). The data of Muchi et al. show that the dispersion coefficients increase regularly with the velocities of solids as well as that of gas.

Generally, from the macro point of view, rodlike flow of solids in a moving bed is assumed. In actual moving beds, however, the solids may move downward with undulation, and the voids alternately expand and contract. Therefore it is supposed that the fluid mixing is affected not by the solids velocity itself but by the pattern of moving solids, which consequently varies with the solids velocity.

Employing the same apparatus and glass beads as used in the experiments described in our previous paper ${ }^{13)}$, an experimental investigation was carried out to measure the longitudinal dispersion coefficients $E_{x}$ to check the effect of the moving solids, and to compare them with the data for packed beds at low Reynolds numbers.

* Received on September 9, 1970

** Mobil Sekiyu Kabushiki Kaisha, Tokyo

\section{Experiments}

According to the authors' paper ${ }^{13)}$, pressure drop through a moving bed is originated by the slip velocity between the gas flow and the descending solids, and the flowing gas measured at the outlet of a bed is not exactly the same as the flowing gas through the test section. With high descending velocity of fine solids, gas may flow downward against the higher pressure at the bottom of the bed. Considering this information, interstitial gas velocities were determined for the various solids velocities under constant pressure drop.

Helium, which was steadily introduced into the middle part of a moving bed of glass beads was stopped instantaneously, and the concentration time curves (I curves) were measured by thermal conductivity cells. Two sets of response curves taken $20 \mathrm{~cm}$ apart were obtained. Errors due to the end effects were thereby eliminated.

\section{Results and Discussion}

After differentiating the I curves numerically, the variances of the concentration distributions were evaluated. If each pair of response curves was obtained at two positions (denoted suffixes 1 and 2) at some distance apart in an infinite bed, the following simple 